${ }^{1}$ Departament of Restorative Dentistry, Piracicaba Dental School, University of Campinas (UNICAMP), Piracicaba, São Paulo, Brazil.

${ }^{2}$ Private clinic.

${ }^{3}$ University Center UniFTC, Salvador, Bahia, Brazil.

${ }^{4}$ Department of Dental Clinic, School of Dentistry, Federal University of Bahia, Salvador, Bahia, Brazil.

*Corresponding author:

Paula Mathias

email: pmathias@yahoo.com

Received: October 15, 2020

Accepted: February 22, 2021

Editor: Dr Altair A. Del Bel Cury

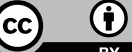

\section{Effect of ceramic thicknesses and opacities on water sorption and solubility of a light-curing resin cement by different units}

\author{
Gabriela Alves de Cerqueira' ${ }^{1}$ iD, Lais Sampaio Souza² (iD, \\ Rafael Soares Gomes ${ }^{3}$ (iD, Giselle Maria Marchi ${ }^{1}$ (iD, \\ Paula Mathias, ${ }^{4 *}$ iD
}

Aim: This study evaluated the water sorption and solubility of a light-cured resin cement, under four thicknesses and four opacities of a lithium disilicate ceramic, also considering three light-emitting diode (LED) units. Methods: A total of 288 specimens of a resin cement (AllCem Veneer Trans FGM) were prepared, 96 samples were light-cured by each of the three light curing units (Valo - Ultradent / Radii-Cal - SDI / Bluephase II - Ivoclar Vivadent), divided into 16 experimental conditions, according to the opacities of the ceramic: High Opacity (HO), Medium Opacity (MO), Low Translucency (LT), High Translucency (HT), and thicknesses $(0.3,0.8,1.5$, and $2.0 \mathrm{~mm})(\mathrm{n}=6)$. The specimens were weighed at three different times: Mass M1 (after making the specimens), M2 (after 7 days of storage in water), and M3 (after dissection cycle), for calculating water sorption and solubility. Results: The higher thickness of the ceramic $(2.0 \mathrm{~mm})$ significantly increased the values of water

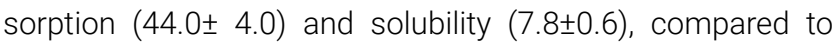
lower thicknesses. Also, the ceramic of higher opacity $(\mathrm{HO})$ generated the highest values of sorption and solubility when compared to the other opacities, regardless of the thickness tested (ANOVA-3 factors / Tukey's test, $a=0.05$ ). There was no influence of light curing units. Conclusion: Higher thicknesses and opacities of the ceramic increased the water sorption and solubility of the tested light-cured resin cement.

Keywords: Cementation. Ceramics. Light-curing of dental adhesives. Resin cements. 


\section{Introduction}

In recent years, minimal intervention dentistry has presented, as an aesthetic restorative alternative, the use of ceramic veneers, with reduced dental preparation ${ }^{1,2}$. Among the ceramic options, lithium disilicate has been widely used because it allows adhesive cementation procedures ${ }^{3}$ and is highly aesthetic, reproducing optical effects similar to those of natural teeth and enabling efficient masking of teeth with chromatic changes ${ }^{4,5}$. Ceramic laminates are fixed to the teeth using adhesive systems and resin cement, which is capable of generating a strong bond between the dental substrate and the laminate ceramic ${ }^{1,5}$.

Resin cement can be classified according to its polymerization: self-curing, light-curing, and dual ${ }^{5,6}$. For ceramic laminate veneers, light-cured cements are the most suitable, since self-curing and dual cements present, in their composition, tertiary amines, which, when reacted with benzoyl peroxide, become responsible for the yellowing of the material ${ }^{6}$. Considering that ceramic laminates have a reduced thickness in many cases, this yellowing could compromise the aesthetic result of the treatment, especially in long term ${ }^{1,7}$.

The ceramic material used in the restorative technique can present different thicknesses and opacities, which are associated with the depth of preparation and the darkness of the tooth². The different thicknesses and opacities of the ceramics directly affect the amount of light available to light-cure the resin cement underlying the ceramic material ${ }^{4,6}$. This is because the light radiated by the light-curing unit must go through the ceramic and reach the cement, in a uniform, efficient, and satisfactory manner, to achieve a high degree of conversion of the resin monomers of the cement, ensuring its mechanical properties ${ }^{5,6}$.

More translucent laminates, as well as less thick ones, tend to facilitate the passage of light through the restoration, allowing a greater amount of light to reach the resin cement below the restoration ${ }^{5,8}$. However, in some clinical situations, it is necessary to mask darkened teeth, using a more opaque and thicker ceramic restorations ${ }^{1,8,9}$. There are no conclusive studies in the literature associating different opacities of ceramics with different thicknesses to determine which situations could ensure the best passage of light through the ceramic restoration; or about which of the two variables - thickness or opacity - is able to compromise this passage of light more significantly15,6,10.

In addition to the optical characteristics and thickness of the ceramic, the parameters of the light must also be considered ${ }^{11}$. A high intensity of power, along with a homogeneous and collimated light beam, are required so that the light is able to pass through the ceramic, without losing so much power and without dissipating too much into the material ${ }^{12}$. The delivery of this light energy to resin cement is also strongly influenced by the time of application of the light ${ }^{2,10}$. In addition, the light must have wavelengths in the absorbance range of the photoinitiators contained in the resinous material, such as camphorquinone, considered the main photoinitiator, which shows its absorption peak around $468-470 \mathrm{~nm}^{2,6,10}$. Thus, the importance of light in the light-curing process of resin cements makes the analysis of the light-curing parameters essential for the safety of clinical procedures. 
When the light curing of resin cement is compromised, some negative effects can occur, such as a low degree of conversion and high water sorption and solubility of the material, which will generate poor mechanical properties, infiltration and detachment of the restoration ${ }^{5,6,11,13}$. In the oral environment, fluid sorption can occur, resulting in the swelling of the resinous material at the tooth interface/restoration, causing changes in its organic matrix and impairment in the structure of this material, because of the dissociation of the inorganic charge. This decreases the resistance and the solubility of the material, reducing its mass by leaching its components and, consequently, releasing unreacted monomers, which can even cause damage to the dental pulp $p^{1,13-16}$.

Thus, it is important to understand the influence of dental ceramic thickness and opacity both alone and in association on water sorption and solubility of resin cement, using different light-curing units. The hypothesis of this study was that the greater the thickness and opacity of the ceramic interposed, the higher the water sorption and solubility values of the resin cement, regardless of the light-curing unit used. Therefore, this study aimed to evaluate the physical properties (water sorption and solubility) of a light-cured resin cement under four thicknesses and four opacities of a lithium disilicate-based ceramic, considering three different light-emitting diode (LED) units.

\section{Materials and methods}

\section{Preparation of Specimens}

A total of 288 specimens of AllCem Veneer Trans resin cement (FGM, Dentscare LTDA, Joinville-SC, Brazil) were prepared (Table 1), being light-cured under four different thicknesses and opacities of a ceramic based on lithium disilicate (IPS e.Max Press - Ivoclar-Vivadent), also considering three different light emitting diode (LED) units. For each of the three light-curing units (Valo - Ultradent, Radii-Cal - SDI, and Bluephase II - Ivoclar Vivadent) tested, 96 specimens were obtained, divided into 16 experimental conditions: four opacities (HT - High Translucency, LT - Low Translucency, MO - Medium Opacity, and HO - High Opacity) and four thicknesses (0.3 / 0.8 / 1.5 / 2 mm) ( $\mathrm{n}=6$ ). G1-HT/0.3mm; G2-HT/0.8mm; G3-HT/1.5 mm; G4-HT/2.0mm; G5-LT/0.3mm; G6-LT/0.8mm; G7-LT/1.5mm; G8-LT/2.0mm; G9-MO/0.3mm; G10$\mathrm{MO} / 0.8 \mathrm{~mm} ; \mathrm{G} 11-\mathrm{MO} / 1.5 \mathrm{~mm} ; \mathrm{G} 12-\mathrm{MO} / 2.0 \mathrm{~mm} ; \mathrm{G} 13-\mathrm{HO} / 0.3 \mathrm{~mm} ; \mathrm{G} 14-\mathrm{HO} / 0.8 \mathrm{~mm}$; $\mathrm{G} 15-\mathrm{HO} / 1,5 \mathrm{~mm} ; \mathrm{G} 16-\mathrm{HO} / 2.0 \mathrm{~mm}$

Table 1. The composition of the light-curing resin cement AllCem Venner Trans (FGM, Dentscare LTDA, Joinville-SC, Brazil)

\begin{tabular}{cc}
\hline Resin Cement & Composition \\
\hline & Methacrylate monomers \\
AllCem Venner Trans & (UDMA, BIS-EMA, BIS-GMA, TEGDMA) \\
Pight-curing & Photoinitiators \\
& Coinitiators \\
Stabilizers & Pigments \\
& Silanized barium-aluminum-silicate glass particles and silicon dioxide \\
\hline
\end{tabular}


All specimens were made using a split stainless steel matrix with 3-mm diameter and 1-mm thickness. On this metallic matrix, filled with a single increment of the resin cement, a polyester strip was placed, followed by a glass coverslip and a weight of $500 \mathrm{mg}$, left for 30 seconds to drain the excess material. Then, the weight and the glass coverslip were removed and the specimen was light-cured for $40 \mathrm{~s}$, through the polyester strip, for the control group. For the other groups, the resin cement was light-cured under a piece of ceramic material that varied its respective opacity and thickness, according to each experimental condition. This sequence was applied, in an identical manner, to the three light-curing units (Valo, Radii-Cal and Bluephase II) (Table 2).

Table 2. Description of light-curing units (LCU) used in this study, considering their respective light parameters (power intensity and wavelength)

\begin{tabular}{lccc}
\hline \multicolumn{3}{c}{ Operation conditions LED devices } \\
\hline & Valo - Ultradent & Radii-Cal - SDI & Bluephase II - Ivoclar Vivadent \\
\hline Light intensity & $1400 \mathrm{~mW} / \mathrm{cm}^{2}$ & $1200 \mathrm{~mW} / \mathrm{cm}^{2}$ & $1200 \mathrm{~mW} / \mathrm{cm}^{2}$ \\
\hline $\begin{array}{l}\text { Wavelength } \\
\text { range }\end{array}$ & $385-515 \mathrm{~nm}$ & $440-480 \mathrm{~nm}$ & $385-515 \mathrm{~nm}$ \\
\hline
\end{tabular}

\section{Evaluation of water sorption and solubility}

After preparation, the specimens were measured using a digital caliper with $0.01 \mathrm{~mm}$ precision (Mitutoyo, Suzano - SP, Brazil). Means of diameter duplicates were calculated for each specimen, to determine the radius ( $r$ ), height ( $h)$, and the individual volume. Following the ISO 4049 specifications ${ }^{17}$, the specimens were placed in a desiccator and transferred to an incubator at $37^{\circ} \mathrm{C}$, for preconditioning. After 24 hours, the specimens were weighed repeatedly, in an interval of 24 hours, until a constant mass (M1) was reached, on an analytical scale (Shimadzu, mod. AUW220D, Barueri - SP, Brazil), with an accuracy of 0.0001 of $1 \mathrm{~g}$. This stabilization was verified when the variation of the values of $\mathrm{M} 1$ was lower than $0.2 \mathrm{mg}$, in a period of $24 \mathrm{~h}$, for each specimen. The specimens were individually identified and stored in an incubator at $37^{\circ} \mathrm{C}$ (Quimis, Diadema - SP, Brazil), being kept in a container with silica gel, for desiccation, for seven consecutive days.

After stabilization of $\mathrm{M} 1$, the specimens were immersed in $2 \mathrm{ml}$ of distilled water $(\mathrm{pH} 7.2)$ and again stored at $37^{\circ} \mathrm{C}$, where they remained for seven consecutive days. Afterwards, the specimens were removed from the distilled water, dried with absorbent paper (Sorella, Canoinhas-SC, Brazil), and weighed again on an analytical scale to obtain the mass (M2). After M2 registration, the specimens were stored individually in the incubator at $37^{\circ} \mathrm{C}$, for desiccation.

All specimens were weighed, repeatedly, at 24h intervals, until a constant mass (M3) was reached, considering a variation lower than $0.2 \mathrm{mg}$ for each specimen.

After acquiring all the mass values of the specimens, water sorption (So) and solubility (Sol) were calculated using the following formulas: 
Where $\mathrm{M} 1$ is the constant mass, in $\mu \mathrm{g}$, found before immersion in water; $\mathrm{M} 2$ is the mass, in $\mu \mathrm{g}$, after immersion in water for 7 days; $M 3$ is the constant mass, in $\mu \mathrm{g}$, after desiccation; and $\mathrm{V}$ is the volume of the specimens in $\mathrm{mm}^{3}$.

\section{Statistical analysis}

The collected data were tabulated and evaluated for their homogeneity and normality, with Levene and Shapiro-Wilk tests being applied, respectively, with a 5\% significance level, for each of the variables (Water sorption and Solubility). Considering the assumptions for the application of the parametric tests, an Analysis of Variance with 3 factors was applied: 1. Light-curing unit in 3 levels (Valo, Radii-cal, and Bluephase); 2. Ceramic thickness in 4 levels $(0.3,0.8,1.5$, and $2.0 \mathrm{~mm})$; and 3 . Opacity in 4 levels (HT - High Translucency, LT - Low Translucency, MO - Medium Opacity, and HO High Opacity). Tukey's test was used as post hoc.

\section{Results}

The statistical analysis showed that the thickness and the degree of translucency of the ceramic interfered in the water sorption and solubility values of the light-cured AllCem resin cement, regardless of the light-curing unit used. As a result of water sorption (Table 3), it was found that the greater thickness of the lithium disilicate ceramic $(2.0 \mathrm{~mm})$ increased the water sorption values when statistically compared to the thicknesses of $0.3 \mathrm{~mm}$ and $0.8 \mathrm{~mm}$. Similarly, medium and high opacity ceramics ( $\mathrm{MO}$ and $\mathrm{HO}$ ) contributed to higher values of water sorption compared to high translucency $(\mathrm{HT})$ ceramics. The higher opacity lithium disilicate $(\mathrm{HO})$ ceramics resulted in higher water sorption values of resin cement than the values showed by high and low translucency ceramics ( $\mathrm{HT}$ and $\mathrm{LT})$. The less thick ceramic $(0.3 \mathrm{~mm})$ with greater opacity $(\mathrm{HO})$ showed increased water sorption values, regardless of the light-curing unit tested. The same behavior was found for high translucency (HT), thick (2.0 mm) ceramic. The type of the light-curing unit tested did not influence the water sorption values of the resin cement, with no statistically significant difference between them.

Table 4 shows the results obtained by analyzing the solubility data of the resin cement. It was found that, as with sorption, the greater thickness of the lithium disilicate ceramic ( $2 \mathrm{~mm}$ ) increased the solubility values, compared to the values obtained with the thickness of $0.3 \mathrm{~mm}$, regardless of the light-curing unit tested. Likewise, the difference for the other thicknesses was statistically significant with the increase in the opacity of the ceramic. The more translucent the ceramics (HT and LT), the lower the solubility values of the resin cement compared to the values obtained with the higher opacity ceramic ( $\mathrm{HO})$, for the three light-curing units tested. Similarly to water sorption, the type of light-curing unit, by itself, did not affect the solubility values, with no statistically significant difference between them. 


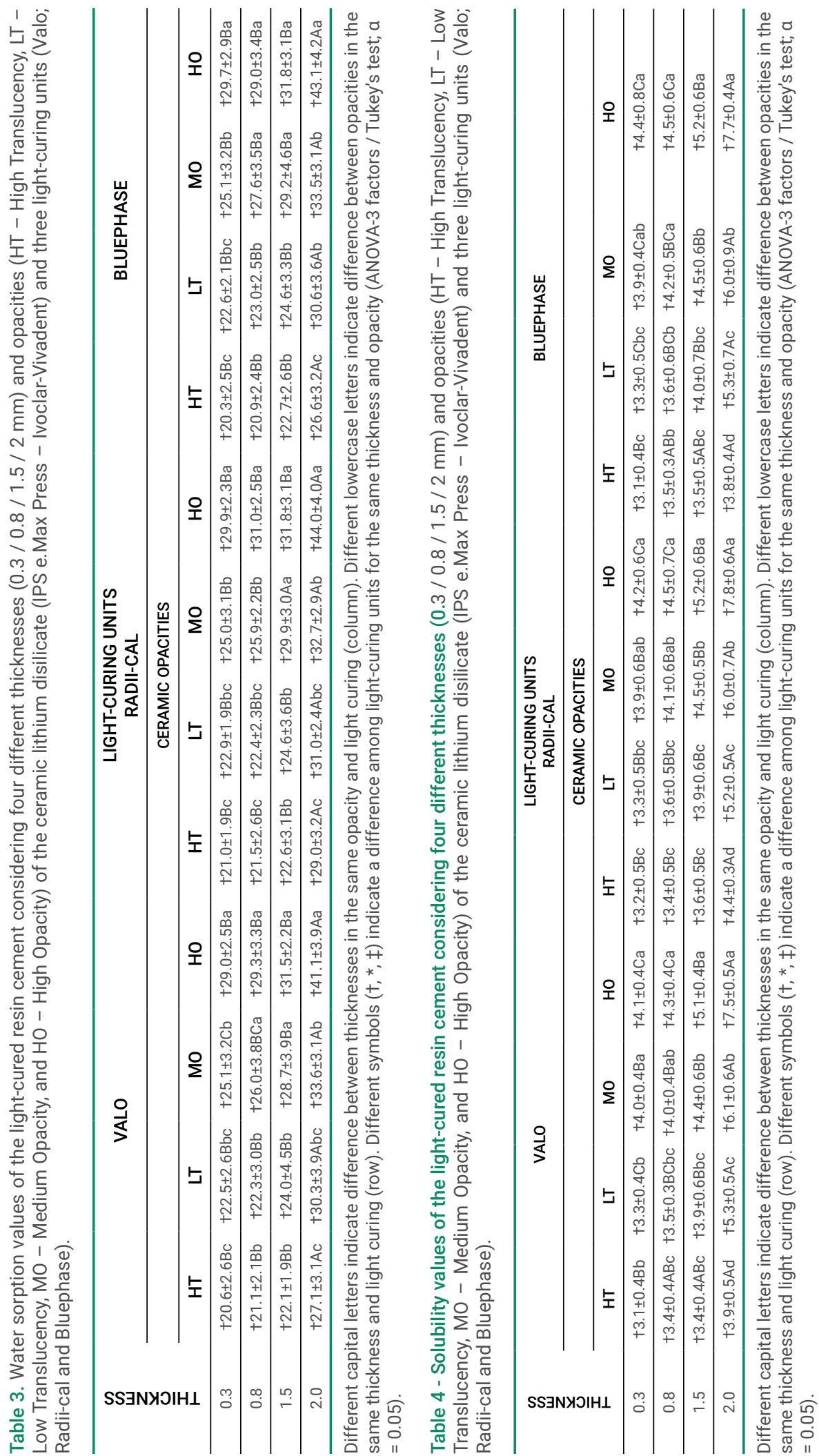




\section{Discussion}

The mechanical properties of resin cement can be affected by the water sorption and solubility that occur in an aqueous environment, such as in the oral environment ${ }^{5}$. Studies show that increasing the thickness and opacity of the ceramic restoration reduces the passage of light during the photoactivation of cement, compromising the performance of the material1,8,10,18. In this study, the experimental hypothesis was partially accepted, since the ceramics of greater thickness and opacity interposed during light-curing procedures increased the values of water sorption and solubility of the resin cement. However, this increase was not gradual as the opacity and/or thickness of the ceramic increased, with similar behaviors being observed among ceramics of different thicknesses. The tested light-curing units were not different.

In our study, thicknesses of 0.3 and $0.8 \mathrm{~mm}$ resulted in similar values to each other and significantly lower for water sorption and solubility, compared to the thickness of $2 \mathrm{~mm}$, for all three light curing units tested. These results corroborate the data reported by Runnacles et al..$^{8}$ (2014), who has shown that the effect of light attenuation by ceramic veneers is not significant in thicknesses up to $1.0 \mathrm{~mm}$. Previous studies have also shown that the thinner the ceramic material interposed between the resin cement and the light source, the greater the degree of conversion of the resin material ${ }^{18,19}$. According to Calgaro et al. $^{20}$ (2013), the increase in thickness is a key factor in attenuating the light emitted by the light-curing unit, since they observed that the polymerization decreases as the thickness increases.

In this study, increasing the thickness of the ceramic to $2.0 \mathrm{~mm}$ also significantly increased the water sorption and the solubility of the resin cement under it. Similar observations were reported in a previous study, where the resin microhardness and roughness was reduced when the thickness of the ceramic increased from $1 \mathrm{~mm}$ to $2 \mathrm{~mm}^{21}$. The explanations for this change in properties of the light-cured resin cement under thicker ceramics may be related to the reduction of the light transmitted by the light-curing unit when crossing a $2 \mathrm{~mm}$ thick ceramic ${ }^{22}$. According to Liebermann et al. ${ }^{23}$ (2018), light transmittance is inversely related to the thickness of the ceramic, which will be crossed by the light beam. That is, the thicker the material, the lower the transmittance of that light ${ }^{22,23}$. Transmittance can be defined as the amount of light that passes through a material, part of which is reflected or absorbed ${ }^{23}$. If a small part of this light is scattered and most of it is transmitted through the material, higher transmittance values will be achieved ${ }^{22,23}$. In a ceramic material, the light ends up being too dispersed and diffusely reflected, generating an opaque appearance ${ }^{22,23}$. It should also be noted that more translucent materials show changes in light transmittance due to the variation in thickness, thus, even translucent materials, when thicker, reduce light transmittance ${ }^{22,23}$. This may explain the fact that the interposition of a translucent ceramic (HT), with a thickness of $2.0 \mathrm{~mm}$, also results in an increase in the values of water sorption and solubility of the light-cured cement under it in this study.

As well as the thickness, the degree of translucency of the ceramic laminate has a strong influence on the polymerization of the resin cement under the ceramic lami- 
nat $^{1,10}$. In this study, when the HT and LT ceramics were used with the same thickness, the resin cement showed the lowest values of water sorption and solubility, for the three light units tested. This influence of the degree of translucency of the ceramic can be explained by the microstructure of the ceramic material, especially its crystalline phase, which tends to present differences in the transmittance and dispersion of light, affecting light transmission and, consequently, the light irradiance that reaches the resin cement underlying the laminate ${ }^{4}$. Leal et al. ${ }^{1}$ (2016) observed that ceramics with lower translucency (more opaque) limit the passage of light emitted by the light-curing units. Likewise, Calgaro et al. ${ }^{20}$ (2013), when testing different types of ceramics, observed that, among the $\mathrm{HT}, \mathrm{LT}$, and $\mathrm{MO}$ ceramics, the best performance in the degree of conversion of the underlying resin cement was achieved by HT ceramics. The results found in our study corroborate these observations, since the resin cement under ceramics with a low level of translucency ( $\mathrm{MO}$ and $\mathrm{HO}$ ) showed greater water sorption and solubility. The less translucent ceramics are often used to mask teeth with severe stains or with significant color differences between substrate and final color of the restoration ${ }^{1,10}$.

When correlating the thickness and translucency variables of the ceramics tested in this study, it can be seen that, regarding thickness for ceramics up to $1.5 \mathrm{~mm}$, in HT and LT opacities, no significant differences were observed for water sorption and resin cement solubility. The increase in the thickness of the ceramic piece to $2.0 \mathrm{~mm}$ resulted in significant increases in sorption and solubility for all degrees of translucency tested, confirming the care with measuring ceramic thickness during adhesive cementation procedures with light-curing resin cements. De Jesus et al. ${ }^{4}$ (2020) also showed that ceramics with thickness higher than $1.5 \mathrm{~mm}$ reduce the values of degree of conversion and microhardness of the resinous material below to ceramic restoration. The association of $2.0 \mathrm{~mm}$ thickness with higher opacities (MO and $\mathrm{HO}$ ) increased the values of water sorption and solubility even more, probably due to the greater impairment of light transmission through the respective ceramic pieces. Concerning the opacity variable, for the $\mathrm{HO}$ ceramic, all thicknesses resulted in higher values of water sorption and solubility, except the minimum thickness of $0.3 \mathrm{~mm}$. HO ceramics with thicknesses of 1.5 and $2.0 \mathrm{~mm}$ make the tested diffusion dynamics values even more critical. Higher values of water sorption and solubility of resin cements under thicker and more opaque ceramic laminates probably come from a lower degree of conversion and less microhardness of these cements, after polymerization $4,9,22,23$. With the increase in the thickness and opacity of the ceramic, there is a decrease in the passage of light to reach the resin cement, reducing its degree of conversion and its microhardness ${ }^{4,9}$.

AccordingtotheAmericanDentalAssociationspecification, thesorptionofresinousmaterials must be less than $40 \mathrm{lg} / \mathrm{mm}^{3}$ and the water solubility must be less than $7.5 \mathrm{lg} / \mathrm{mm}^{3}$, for a storage period of seven days ${ }^{24}$. In this study, when the resin cement was light-cured under the 2-mm thick ceramic piece with high opacity $(\mathrm{HO})$, it exceeded this acceptable water sorption limit for the three light-curing units tested: Valo $\left(41.1 \mathrm{lg} / \mathrm{mm}^{3}\right)$, Radical $\left(44.0 \mathrm{lg} / \mathrm{mm}^{3}\right)$, and Bluephase $\left(43.1 \mathrm{lg} / \mathrm{mm}^{3}\right)$. The same occurred for the solubility, with values above the limit for all LED units: Radical $\left(7.8 \mathrm{lg} / \mathrm{mm}^{3}\right)$, Bluephase $\left(7.7 \mathrm{lg} / \mathrm{mm}^{3}\right)$, and Valo $\left(7.5 \mathrm{lg} / \mathrm{mm}^{3}\right)$. Therefore, the association of high 
opacity with a $2.0 \mathrm{~mm}$ ceramic tile thickness demonstrates a detrimental effect on the physical properties of diffusion dynamics of resin cement, and should be considered in clinical cementation procedures. In their study, Leal et al. ${ }^{1}$ (2016) identified mean values higher than the acceptable limit both for water sorption and solubility of light-cured resin cement, when ceramic pieces of medium and low translucency were positioned on the resin cement. On the other hand, when highly translucent surfaces are used, values similar to the control conditions were found, indicating no significant influence to light transmission, which allowed an adequate conversion of resin monomers ${ }^{25,26}$. When a light-curing material does not receive the appropriate amount of light energy, an impaired formation of free radicals that initiate polymerization and a lower degree of conversion of the polymer network are observed ${ }^{27}$.

The resin cement used in this study has low viscosity, and, in its composition, high and low molecular weight monomers (Table 1). It is known that the composition of the organic matrix of resin cements can influence water sorption and solubility 5 . Lower molecular weight monomers, such as TEGDMA (triethylene glycol dimethacrylate) or UDMA (urethane dimethacrylate), are mixed with higher molecular weight monomers, such as Bis-GMA (bisphenol A-diglycidyl dimethacrylate), to promote less viscosity, changing the material handling properties and collaborating with the technical cementation procedures ${ }^{5,13}$. However, monomers of lower molecular weight also have a more hydrophilic nature, which may allow greater diffusion dynamics of resin cements ${ }^{28-30}$. The hydrophilicity of these monomers can generate undesirable clinical consequences, such as microleakage, susceptibility to degradation, discoloration and decreased mechanical properties, postoperative sensitivity, and recurrent caries ${ }^{1,8,31}$. It is believed that water gain is related to the composition of the material, the content, concentration and type of inorganic fillers, as well as the size and nature of the particles ${ }^{1}$. Therefore, the water sorption and solubility values of the resin cement used in this study may also have been influenced by the hydrophilicity characteristics of the monomers incorporated in its matrix. In our study, the behavior of this cement was not compared to that of other cements, so that a more accurate assessment of the influence of this composition could be considered. Therefore, in future research, comparative investigation between different cements is recommended.

In this study, three high power light-curing units were tested, with no significant difference between them. Light intensity, irradiance, and light application time are factors that may be associated with the conversion of resin monomers from resinous material $^{18}$. For some authors, variations in the power of the light emitting device used can directly affect the mechanical properties of the material, showing the need to work at maximum intensity ${ }^{1}$. It should be noted, however, that the use of high energy densities, per se, is not directly related to higher degrees of conversion ${ }^{32}$. Other factors should be considered, such as the use of a light-curing unit with an appropriate wavelength, to sensitize photoinitiators to the resin material used ${ }^{33}$. In view of the need for the emergence of clearer resin materials for cosmetic procedures in dentistry, alternative photoinitiators were inserted in their composition, changing the peak wavelength absorption used for camphorquinone ${ }^{34}$. Therefore, some LED units were created with polywave technology, which allows them to emit a broader wavelength spectrum, photosensitizing all the photoinitiators present in the composition of the 
material, unlike monowave LED units, which normally only reach camphorquinone, since they have a more limited spectrum ${ }^{34,35}$. In this study, two of the light-curing units tested are considered polywave (Bluephase and Valo). However, the absence of difference between them can be justified by the composition of the resin cement, which has a photoinitiator compatible with the wavelength spectrum emitted for all three light-curing units. This highlights the need for including different resinous materials, with different compositions and photoinitiators, in future studies.

In addition, the presence of a collimated light beam seems to have a considerable influence on the light-curing of resinous materials, especially when a barrier is placed between the light source and the resinous material, as in the case of ceramic restorations $^{12,33}$. The linear orientation of the light beams prevents this light from dispersing, which favors the delivery of energy density to the restorative material ${ }^{12,33}$. Considering the exposure time factor, Archegas et al. ${ }^{25}$ (2012) observed that the polymerization of resin cements for 40 s through an opaque ceramic resulted in a lower degree of conversion than through a translucent ceramic. Also, a time of 120 s resulted in similar degree of conversion values for opaque and more translucent ceramics ${ }^{25}$. Uctasli et al..$^{10}$ (1994) found that the use of a thicker and more opaque ceramic requires greater exposure to irradiated light, but even so, there is a limit of thickness and opacity so that the appropriate polymerization is obtained at the best irradiation conditions. The authors emphasized that the photoactivation time should not be less than $40 \mathrm{~s}$. In this study, the exposure time used was standardized at 40s, for all three light-curing units tested, and there was no statistically significant difference among them.

Based on the results found in the present study, the factors opacity and thickness significantly affected the water sorption and solubility of the light-cured resin cement underlying the ceramics; therefore, they need to be evaluated by the professional. Often, ceramic pieces have variable thicknesses and need to be measured and evaluated when opting for adhesive cementation with light-curing resin cements, since the use of a ceramic restoration may not ensure effective light transmission through it, if its thickness is equal or greater to $2.0 \mathrm{~mm}$. Another factor that must be considered is the light-curing unit used, which must have adequate characteristics, especially regarding a high intensity of emitted power, for effective photopolymerization.

In conclusion, the light-curing resin cement under lithium disilicate ceramics with a thickness equal to or greater than $2.0 \mathrm{~mm}$ and/or high opacity presents high water sorption and solubility. The greater degree of opacity $(\mathrm{HO})$ increased values of water sorption and solubility of resin cement in all tested thicknesses, regardless of the high-power light curing units tested.

\section{Acknowledgments}

The authors thank Espaço da Escrita - Dean of Research - UNICAMP for the language services provided. We also thank the Institutional Scientific Initiation Scholarship Program (PIBIC), the Bahia Research Foundation (FAPESB), and the National Council for Scientific and Technological Development (CNPq) for the financial support given to this study. 


\section{References}

1. Leal CL, Queiroz APV, Foxton RM, Argolo S, Mathias P, Cavalcanti AN. Water sorption and solubility of luting agents used under ceramic laminates with different degrees of translucency. Oper Dent. 2016;41(5):E141-8. doi: 10.2341/15-201-L.

2. Rasetto FH, Driscoll CF, Von Fraunhofer JA. Effect of light source and time on the polymerization of resin cement through ceramic veneers. J Prosthodont. 2001 Sep;10(3):133-9. doi: 10.1111/j.1532849x.2001.00133.x.

3. Mobilio N, Fasiol A, Catapano S. Qualitative evaluation of the adesive interface between lithium disilicate, luting composite and natural tooth. Ann Stomatol (Roma). 2016 Jul;7(1-2):1-3. doi: 10.11138/ads/2016.7.1.001.

4. de Jesus RH, Quirino AS, Salgado V, Cavalcante LM, Palin WM, Schneider LF. Does ceramic translucency affect the degree of conversion of luting agents? Appl Adhes Sci. 2020;8(1):2-10. doi: 10.1186/s40563-020-00127-2.

5. Queiroz APMV, Queiroz MMV, Argolo S, Foxton RM, Mathias P, Cavalcanti AN. Effect of the ceramic translucency on the long-term water sorption and solubility of resin cements. Brazilian J Oral Sci. 2020;19:e208224. doi: 10.20396/bjos.v19i0.8658224.

6. Jung H, Friedl KH, Hiller KA, Furch H, Bernhart S, Schmalz G. Polymerization efficiency of different photocuring units through ceramic discs. Oper Dent. 2006;31(1):68-77. doi: 10.2341/04-188.

7. Peumans M, de Munck J, Fieuws S, Lambrechts P, Vanherle G, Meerbeek BV. A prospective tenyear clinical trial of porcelain veneers. J Adhes Dent. 2004 Spring;6(1):65-76.

8. Runnacles P, Correr GM, Baratto Filho F, Gonzaga CC, Furuse AY. Degree of conversion of a resin cement light-cured through ceramic veneers of different thicknesses and types. Braz Dent J. 2014;25(1):38-42. doi: 10.1590/0103-6440201302200.

9. Soares CJ, Silva NR, Fonseca RB. Influence of the feldspathic ceramic thickness and shade on the microhardness of dual resin cement. Oper Dent. 2006;31(3):384-9. doi: 10.2341/05-51.

10. Uctasli S, Hasanreisoglu U, Wilson HJ. The attenuation of radiation by porcelain and its effect on polymerization of resin cements. J Oral Rehabil. 1994 Sep;21(5):565-75. doi: 10.1111/j.13652842.1994.tb01171.x.

11. Thonemann B, Schmalz G, Hiller KA, Schweikl H. Responses of L929 mouse fibroblasts, primary and immortalized bovine dental papilla-derived cell lines to dental resin components. Dent Mater. 2002 Jun;18(4):318-23. doi: 10.1016/s0109-5641(01)00056-2.

12. Rueggeberg FA, Giannini M, Arrais CAG, Price RBT. Light curing in dentistry and clinical implications: a literature review. Braz Oral Res. 2017 Aug;31(suppl 1):e61. doi: 10.1590/1807-3107BOR-2017. vol31.0061.

13. Mese A, Burrow MF, Tyas MJ. Sorption and solubility of luting cements in different solutions. Dent Mater J. 2008 Sep;27(5):702-9. doi: 10.4012/dmj.27.702.

14. Gerdolle DA, Mortier E, Jacquot B, Panighi MM. Water sorption and water solubility of current luting cements: An in vitro study. Quintessence Int. 2008 Mar;39(3):e107-14.

15. Malacarne J, Carvalho RM, de Goes MF, Svizero N, Pashley DH, Tay FR, et al. Water sorption/ solubility of dental adhesive resins. Dent Mater. 2006 Oct;22(10):973-80. doi: 10.1016/j. dental.2005.11.020.

16. Toledano M, Osorio R, Osorio E, Fuentes V, Prati C, Garcia-Godoy F. Sorption and solubility of resinbased restorative dental materials. J Dent. 2003 Jan;31(1):43-50. doi: 10.1016/s03005712(02)00083-0. 
17. International Standard Organization. ISO 4049:2009. Dentistry - Polymer-based restorative materials. ISO; 2009.

18. Martins FV, Vasques WF, Fonseca EM. How the variations of the thickness in ceramic restorations of lithium disilicate and the use of different photopolymerizers influence the degree of conversion of the resin cements: a systematic review and meta-analysis. J Prosthodont. 2019 Jan;28(1):e395-e403. doi: 10.1111/jopr.12920.

19. Lee JW, Cha HS, Lee JH. Curing efficiency of various resin-based materials polymerized through different ceramic thicknesses and curing time. J Adv Prosthodont. 2011 Sep;3(3):126-31. doi: 10.4047/jap.2011.3.3.126.

20. Calgaro PAM, Furuse AY, Correr GM, Ornaghi BP, Gonzaga CC. Influence of the interposition of ceramic spacers on the degree of conversion and the hardness of resin cements. Braz Oral Res. 2013;27(5):403-9. doi: 10.1590/S1806-83242013000500004.

21. Jafari Z, Alaghehmand $H$, Samani $Y$, Mahdian M, Khafri S. Light transmittance of CAD/CAM ceramics with different shades and thicknesses and microhardness of the underlying light-cured resin cement. Restor Dent Endod. 2018 Jun;43(3):e27. doi: 10.5395/rde.2018.43.e27.

22. Stawarczyk B, Awad D, Ilie N. Blue-light transmittance of esthetic monolithic CAD/CAM materials with respect to their composition, thickness, and curing conditions. Oper Dent. 2016;41(5):531-40. doi: 10.2341/15-252-L.

23. Liebermann A, Freitas Rafael C, Colle Kauling AE, Edelhoff D, Ueda K, Seiffert A et al. Transmittance of visible and blue light through zirconia. Dent Mater J. 2018 Sep 30;37(5):812-7. doi: 10.4012/ dmj.2016-287.

24. American National Standard/American Dental Association specification no. 27 for resin-based filling materials. Chicago: American Dental Association, Council on Scientific Affairs; 1993

25. Archegas LRP, de Menezes Caldas DB, Rached RN, Soares P, Souza EM. Effect of ceramic veneer opacity and exposure time on the polymerization efficiency of resin cements. Oper Dent. 2012;37(3):281-9. doi: 10.2341/11-134-L.

26. Koch A, Kroeger M, Hartung M, Manetsberger I, Hiller k, Schmalz G et al. Influence of ceramic translucency on curing efficacy of different light-curing units. J Adhes Dent. 2007 Oct;9(5):449-62.

27. Wambier L, Malaquias T, Wambier DS, Patzlaff RT, Bauer J, Loguercio AD et al. Effects of prolonged light exposure times on water sorption, solubility and cross-linking density of simplified etchandrinse adhesives. J Adhes Dent. 2014 Jun;16(3):229-34. doi: 10.3290/j.jad.a32034.

28. Alshalia RZ, Salimc NA, Satterthwaitea JD, Silikas N. Long-term sorption and solubility of bulk-fill and conventional resin-composites in water and artificial saliva. J Dent. 2015 Dec;43(12):1511-8. doi: 10.1016/j.jdent.2015.10.001.

29. Muller JÁ, Rohr N, Fischer J. Evaluation of ISO 4049: water sorption and water solubility of resin cements. Eur J Oral Sci. 2017 Apr;125(2):141-50. doi: 10.1111/eos.12339.

30. Reis AF, Giannini M, Pereira PNR. Influence of water-storage time on the sorption and solubility behavior of current adhesives and primer/adhesive mixtures. Oper Dent. 2007;32(1):53-9. doi: 10.2341/06-13.

31. Chaves P, Graciano FMO, Bim Junior O, Pedreira APRV, Manso AP, Wang L. Water interaction with dental luting cements by means of sorption and solubility. Braz Dent Sci. 2013;15(4):29-35. doi: 10.14295/bds.2012.v15i4.826.

32. Calheiros FC, Braga RR, Kawano Y, Ballester RY. Relationship between contraction stress and degree of conversion in restorative composites. Dent Mater. 2004 Dec;20(10):939-46. doi: 10.1016/j. dental.2004.03.003.

33. Price RBT. Light curing in Dentistry. Dent. Clin. North Am. 2017 Oct;61(4):751-78. doi: 10.1016/j. cden.2017.06.008. 
de Cerqueira et al

34. de Oliveira DCRS, Rocha MG, Correa IC, Correr AB, Ferracane JL, Sinhoreti MAC. The effect of combining photoinitiator systems on the color and curing profile of resin-based composites. Dent Mater. 2016 Oct;32(10):1209-17. doi: 10.1016/j.dental.2016.06.010.

35. Jandt KD, Mills RW. A brief history of LED photopolymerization. Dent Mater. 2013 Jun;29(6):605-17. doi: 10.1016/j.dental.2013.02.003. 